\title{
Prolonged AIDS-free Survival for SIV-infected Macaques Treated With Anti-FasL
}

\author{
Maria S Salvato*¥1, C Cameron Yin 2,3, Hideo Yagita ${ }^{3}$, Toshihiro Maeda ${ }^{4}$, \\ Ko Okumura ${ }^{4}$, Ilia Tikhonov ${ }^{1}$ and C David Pauza ${ }^{1}$
}

\author{
Address: ${ }^{1}$ Institute of Human Virology, University of Maryland Biotechnology Institute, Baltimore, MD 21201, ${ }^{2}$ Department of Pathology, \\ University of Wisconsin Medical School, Madison, WI, ${ }^{3}$ Department of Immunology, Juntendo University School of Medicine, Tokyo, Japan and \\ ${ }^{4}$ The Chemo-Sero Therapeutic Research Institute (Kaketsuken), Kumamoto, Japan \\ Email: Maria S Salvato* - msalvato@facstaff.wisc.edu \\ * Corresponding author $\ddagger$ Presenting author
}

from 2005 International Meeting of The Institute of Human Virology

Baltimore, USA, 29 August - 2 September 2005

Published: 8 December 2005

Retrovirology 2005, 2(SuppI I):SI53 doi:10.1 I86/I742-4690-2-SI-SI53

Acute SIV infection of macaques is a model for human AIDS typified by high levels of plasma viremia that decline with the onset of specific viral immunity. SIV infection limits the development of viral immunity and persists to allow for the establishment of chronic progressive disease. The destruction of CD4+ and CD4- lymphocytes argues that both direct and indirect killing mechanisms contribute to the loss of cells. Although early intervention with antiretroviral drugs reduces viremia and disease, it is still unclear whether protection is due to diminished viral cytopathicity or due to blocking a host mechanism for cell killing that is triggered by the high level of viral replication. We tested the role of FasL killing of bystander lymphocytes by injecting monkeys with a humanized monoclonal anti-FasL. Treatment with antiFasL during acute infection reduced the level of apoptosis of circulating T and B cells, peak vRNA levels were unaffected but higher antibody and CTL responses to viral proteins lead to lower set-point viremia among treated macaques. Reduced bystander killing and increased viral immunity were associated with attenuated SIV disease and a significant increase in the life span of infected macaques after transient treatment with a monoclonal antibody against FasL. 